\title{
Equipping scientists for the new biology
}

\author{
Ruedi Aebersold, Leroy E. Hood, and Julian D. Watts
}

A cornerstone of the US research university and public funding of scientific research has been the close link between science education and training, and research. This system has operated under the assumption that the most meritorious research programs also provide the best training ground for new researchers. Though successful to date, this system may be unable meet the challenges presented by the recent emergence of a new technologydriven approach to biological and biomedical sciences. To date, university-based research programs have been carried out by small, autonomous, highly specialized and hypothesis-driven groups. Each focuses on a fairly narrow range of topics, employing a limited range of technologies. Studies reaching beyond the scope of such groups are addressed in the form of collaborations. This structure has rendered the typical research group unable to react quickly to major technological advances or changes in scientific thinking.

The human genome project has catalyzed a new research method we term discovery science. Discovery science, exemplified by genome sequencing projects, enumerates the elements of a system irrespective of any hypotheses on how the system functions. As illustrated by the revolution in yeast biology and genetics as a result of knowing the complete genomic sequence, discovery science is fundamentally changing how hypothesis-driven science can be conducted. Discovery science requires largescale facilities for genome-wide analyses, including DNA sequencing, gene expression measurements, and proteomics. As it generates data on scales of complexity and volume unprecedented in biological sciences, defying analysis by normal means of interpretation, presentation, and publication, discovery science depends on the integration of computational tools to store, model, and disseminate these exploding cascades of information. Discovery science has given rise to, and is a critical part of, a new approach to biology we term systems biolo-

Ruedi Aebersold (ruedi@u.washington.edu) is professor and Julian D. Watts is research scientist at the department of molecular biotechnology, Box 357730, University of Washington, Seattle, WA 98195, and Leroy $E$. Hood is the president and director of the Institute for Systems Biology, 4225 Roosevelt Way NE, Seattle, WA 98105. gy, which involves the studying of all the elements in a biological system both before and after chemical or genetic perturbation. Ultimately, systems biology aims to establish computational models that are predictive of the behavior of the system or its emergent properties in response to any given perturbation.

The challenges of both discovery science and systems biology include their availability to academic institutions, their integration with hypothesis-driven research programs, and their synthesis into an integrated research strategy. The ability of research universities to establish systems biology programs without compromising their strength in hypothesis-driven research is essential for three reasons. First, researchers must be trained in state-of-the-art facilities for universities to continue in their role of science education and training. Second, universities will need such facilities for data generation to fuel their hypothesis-driven research programs. Systems biology and hypothesis-driven research are complementary, with hypothesis-driven research providing the testing ground for systems biology-derived hypotheses. Finally, as discovery science and systems biology are rapidly being adopted by the private sector, universities need to adopt them both to remain competitive, and to guarantee public access to the large information databases generated. As systems biology necessitates facilities on a scale incompatible with the space and resource constraints of traditional university departments, two issues must first be addressed: administration and funding.

Systems biology programs do not fit into a traditional departmental structure, as they are both space-intensive and interdisciplinary, requiring, for example, elements of chemistry, biology, engineering and computer science. To manage programs of such scale and diversity, universities must create new administrative bodies outside and independent of their departmental structure to ensure their systems biology programs remain flexible to respond swiftly to technological innovations. It is vital that all concerned realize that with the rapidly expanding nature of the new research environments now upon us, that decisive actions are required to ensure that the universities continue to remain competitive and at the research forefront.

To circumvent the high cost of systems biology ventures, it seems logical to seek university-private sector partnerships to support their set-up and operational cost, and to complement public funding programs already in place their maintenance. In such a partnership, the university would provide basic research, technology development, education, and training, overseen by faculty members with expertise in appropriate areas of discovery science and systems biology. The private sector would provide financial support, and from their experience of large-scale research, specific technological support. As the private sector currently expends little effort on basic technology development and education, the return for their investment would be suitably trained personnel, and technologies amenable to immediate integration into their existing research and development programs. Without such investment, the private sector will soon reach a point where university research/training programs no longer provide them with suitably qualified and trained personnel, resulting in a need to fund their own staff training programs, a process far more costly than having universities do it for them.

At a time when the private sector is experiencing record profits, such university-private sector arrangements would be seen as an act of social conscience and responsibility. However, once the money is raised, care should be taken to protect university and other public-sector programs thus funded from undue influence by the private sector. In particular, we must ensure that the private sector does not regard such collaborations simply as a source of lower cost data production or research and development. Therefore all funds need to be with "no strings attached".

One would hope that both universities and the private sector appreciate what each would gain from such partnerships, and thus be mindful of preserving the independence of these research programs. Even so, one could envisage a role for legislative bodies to guarantee the independence of such institutions from private sector control, and if required, possible introduction of legislation requiring corporate investment in public research and development programs as a "cost of doing business". We believe that if these important issues are adequately addressed in a timely fashion, scientific research will continue to provide the varied benefits it currently affords society well into the next century. 